\title{
Beadex affects gastric emptying in Drosophila
}

\author{
Cell Research (2014) 24:636-639. doi:10.1038/cr.2014.24; published online 25 February 2014
}

\section{Dear Editor,}

The ingestion and digestion of food is a universal aspect of animal growth and survival and is important for our understanding of obesity and diabetes. All animals have a digestive system that comprises of an upper gastrointestinal tract mainly for the ingestion and storage of food, and a lower gastrointestinal tract or the gut for digestion and absorption. When food is ingested, peristaltic contraction is initiated and directs the bolus through the esophagus toward the stomach or an equivalent structure [1]. In addition to serving as the major site for digestion, the stomach acts as a reservoir to store food. This adaptation may have significance for the survival of many animals in which the immediate access to the infrequent availability of food necessitates large meal consumptions that can then be slowly digested or utilized at a later time. Once the food is triturated in the stomach, it is released into the lower gut where the main function of digestion and absorption of nutrients is carried out. To ensure the efficient extraction of nutrients in the lower gut, gastric emptying of liquefied food from the stomach into the lower gastrointestinal tract must be regulated and coordinated with the status of digestion and absorption. The release of nutrients from early phase of digestion such as complex carbohydrates, fats, and proteins, provides an inhibitory feedback signal to slow the rate of gastric emptying [1]. In mammals, it is known that these nutrient signals in turn regulate the secretion of gastrointestinal hormones and peptides to modulate gastric emptying $[2,3]$. This negative feedback allows ample time for the lower gut to complete the process of digestion and absorption. A major question, however remains to be unclear is how the completion of digestion and absorption in the gut is communicated to the upper gastrointestinal tract to stimulate gastric emptying. It is possible that digestion product(s) associated with the late stage of digestion and absorption may serve as the trigger for the process. To better understand these processes, we turn to the fly as a genetically tractable model system to dissect the function and physiology of digestion and absorption. Both flies and humans have been shown to share surprising conservation in major cellular and molecular pathways, particularly with many metabolic pathways in common [4]. Fundamentally, they also share similarities in their behavioral and physiological responses to eating. First, numerous studies in insects and Drosophila have shown that they possess anatomically analogous gastrointestinal structures that carry out similar functions to those in higher organisms. For example, the Drosophila foregut or the esophagus is connected to an expandable sac-like structure called the crop, which has been thought to serve as a storage organ for ingested food (Figure 1B). Enveloped by visceral muscles that behave similarly to vertebrate smooth muscle, the crop is capable of contraction and peristaltic movements reminiscent of the human stomach. Similar to the small intestine in mammals, the fly midgut is the site of digestion and absorption, which is connected with the hindgut, a structure equivalent to the colon in mammals for water absorption. Second, eating behavior and postprandial gastrointestinal functions in Drosophila have been shown to be influenced by both endocrine and neural pathways, which have homologous components that function similarly in humans [5]. However, the underlying cellular and molecular mechanism regulating gastrointestinal functions is largely unknown.

We report here a genetic screen in Drosophila for mutants that are defective in gastric emptying. We reasoned that defects in gastric motor or sensory function or the stimulus signaling, as in humans, would lead to a dysfunction in gastric emptying. An easily identifiable phenotype would be an enlarged abdomen as a result of the accumulation of food in the crop and the failure to empty into the midgut. Indeed, a recent characterization of the drop-dead $(d r d)$ mutant with a bloated abdomen phenotype revealed that it has defective gut function in crop emptying and associated reduced rate of defecation [6]. However, since crop contraction was not affected, it was unclear what the underlying basis for phenotype is. In our screen we have identified mutants with bloated abdomens.

Here we focus on the initial characterization of one of the candidate mutants, A523, which exhibits abnormal gastric emptying from the crop (Figure 1A and 1B). Mapping analysis placed the mutation on the $\mathrm{X}$ chromosome to a region defined by cytological bands $17 \mathrm{C} 2-$ 

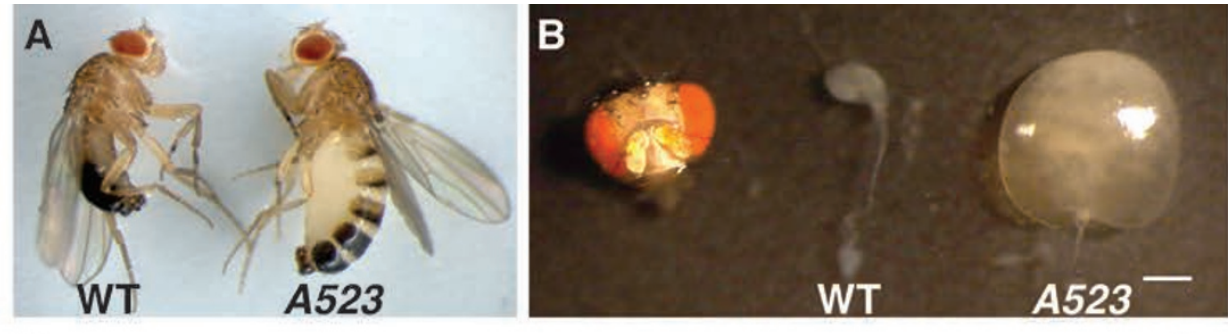

c

D

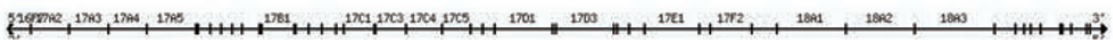
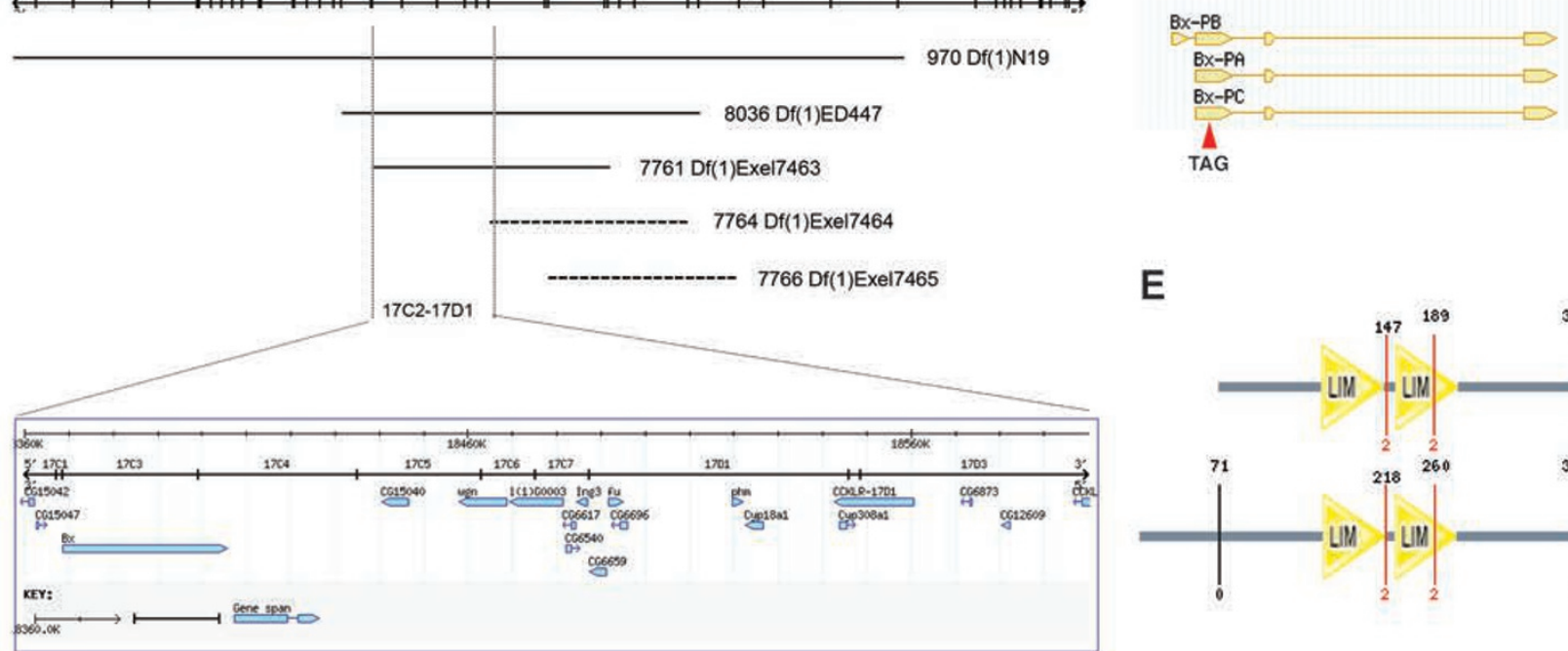

E
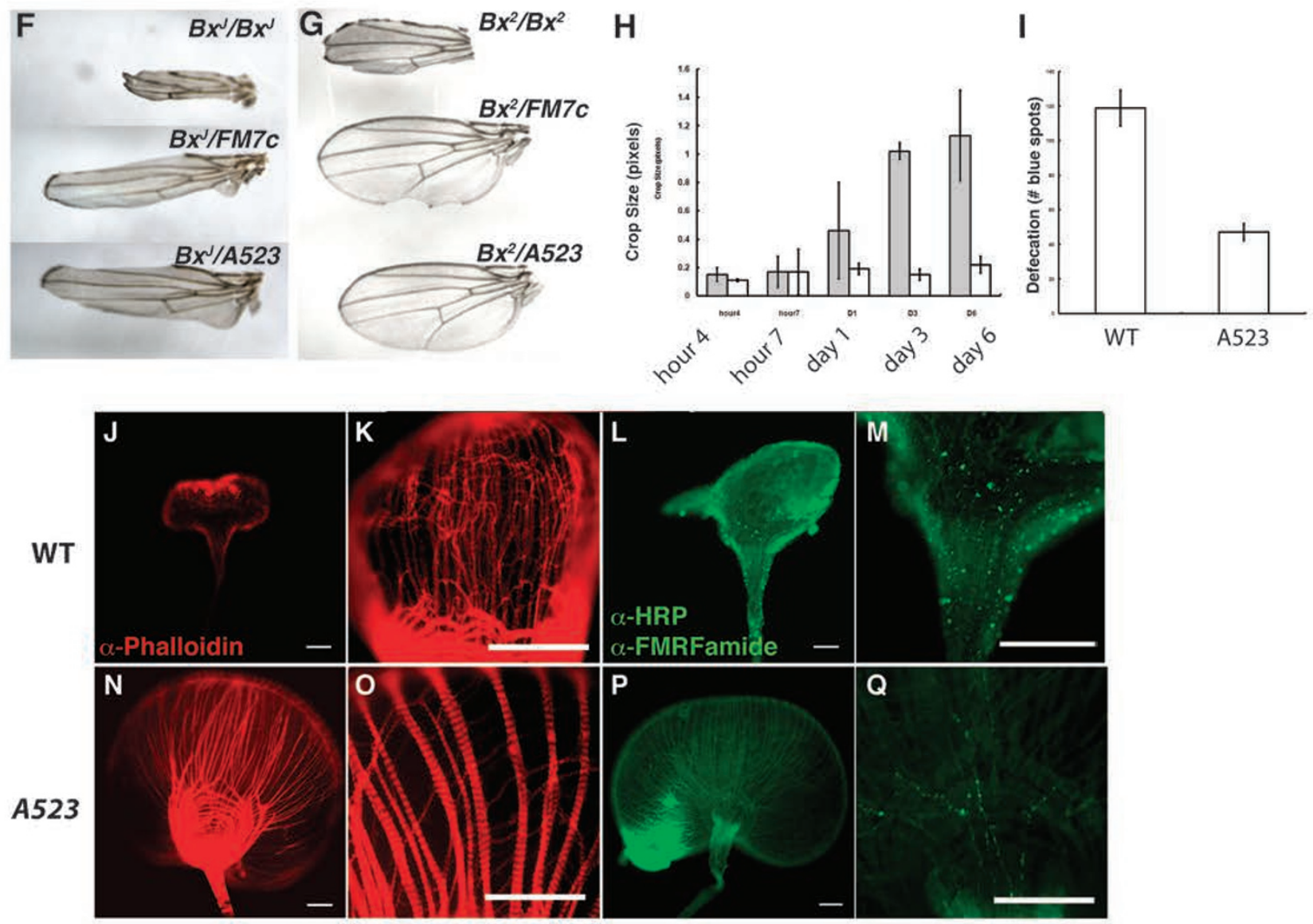
17D1 (Figure 1C). Sequencing of the mutant genomic DNA revealed a single point mutation that introduces an early termination codon in the coding sequence of the Beadex $(B x)$ gene, which encodes the dLMO transcription factor containing two conserved LIM domains [7, 8] (Figure 1D and 1E). The gene is known to be involved in a wide range of development and behavior phenotypes [7, 8]. To demonstrate that $A 523$ is indeed a loss-of-function allele of $B x$, we show that the $A 523$ mutation was able to effectively suppress the dominant wing phenotype of Beadex mutants, which are gain-of-function alleles of the gene $[8,9]$ (Figure $1 \mathrm{~F}$ and $1 \mathrm{G}$ ).

The A523 mutant adults acquire a bloated abdomen as a result of progressive food accumulation in the crop (Figure 1H). This phenotype is apparent one day after eclosion. The enlarged crop phenotype is not due to progressive excessive food intake, but rather correlated with a significant reduction in defecation indicating a defect in gastric emptying (Figure 1I and data not shown). To begin understanding the basis for these defects, we examined the crop structure for any abnormality in muscle function. Phalloidin staining, which highlights muscle fibers, show that the muscles ensheathing the crop differentiate and pattern normally (Figure $1 \mathrm{~J}, 1 \mathrm{~K}, 1 \mathrm{~N}, 1 \mathrm{O}$ ). Although the crop is fully enlarged for a long period of time, there is no indication of the loss of muscle integrity such as broken fibers.

It is known that the crop contraction is affected by numerous neuropeptides secreted by innervating neurons. We asked whether the $A 523$ crop phenotype arises as a result of a defect in such signaling. Staining with antiHRP, which outline the neurons of the crop, and also with anti-FMRFamide, which detects the expression of the crop contraction-inducing neuropeptide FMRFamide, revealed that the neural pathway innervating the crop, at least with respect to FMRFamide, appears to be fully present (Figure 1L, 1M, 1P, 1Q).

The Drosophila esophagus is directly connected to the proventriculus of the midgut while the crop is connected to the esophagus just anterior to the proventriculus. It is not known whether ingested food directly passes into the midgut from the esophagus and excess amount of ingest- ed food is diverted into the crop for storage. Alternatively, all ingested food has to initially enter the crop and in subsequently pumped into the midgut for digestion. The enlarged crop phenotype observed in the $B x$ and $d r d$ mutants could be due to excessive diversion of food into the crop for storage or a defect in the transfer of food from the crop to the midgut. To distinguish these possibilities, we examined the passage of food through the digestive system by following labeled ingested food in adult animals (Supplementary information, Data S1). Our results show that ingested food does not enter directly into the midgut, but rather is initially diverted to the crop during ingestion and subsequently pumped into the midgut after ingestion. This suggests that the crop plays a critical role in regulating food passaging. The fact that $A 523$ and $d r d$ mutants are defective in transferring food out of the crop and show reduced fecal deposition provides further support for this role and indicates that these genes are important for this process [6].

In conclusion, our genetic screen demonstrates the feasibility of using a fly model to identify candidate mutants that affect gastrointestinal function. Furthermore, the crop plays a critical role in gastric emptying. Proteins containing LIM domains have been found to play important roles in many biological processes such as cytoskeleton organization, cell specification and differentiation, as well as organ development and pathological functions [10]. Further studies of $B x$ using tissue-specific Gal4 drivers for different parts of the digestive and nervous systems will hopefully shed light on the mechanism of its physiological regulation of processes such as gastric emptying.

\section{Acknowledgments}

We thank Duc Nguyen (Yale University School of Medicine) for critical reading and editing of the manuscript. This work was supported by grants from the National Basic Research Program of China (973 Program, 2013CB945301) and NIH/NCI to TX, and grants from the National Natural Science Foundation of China $(81170789,81270763)$ and Shanghai Municipal Science and Technology Commission (12431900100) to XW. TX is a Howard Hughes Medical Institute Investigator.

Figure 1 Identification and characterization of a mutant defective in gastric emptying. (A) Adult A523 mutant displayed a bloated abdomen. Animals are 3-4 days old, except where indicated. (B) Dissection revealed an enlarged crop in the mutant as compared to wild type with size reference to the adult head. (C) Deficiencies mapping uncovered the mutation to cytological region 17C2-17D1. (D) Sequencing revealed a single point mutation in the $d L M O$ gene carried by the $A 523$ mutant. (E) Alternatively, spliced dLMO transcripts encoded two proteins with the same $C$-terminal regions containing two LIM domains. (F, G) $B x^{J}$ and $B x^{2}$ wing phenotypes can be suppressed in trans-heterozygotes with A523. (H) A523 mutants show a progressively enlarged crop size apparent after 1 day post eclosion. (I) A523 mutants show a reduction in fecal deposition. (J-O) Phalloidin stainings of the crops of wild type (J-K) and A523 mutant (N-O). (L-Q) Anti-HRP and anti-FMRFamide stainings to highlight neurons and neuropeptide-expressing cells, respectively. (L-M) wild type. (P-Q) A523 mutant. The signals are detected in both wild type and mutant crops, indicating that FMRFamide secretion is present in the mutant. 
Jing Ren ${ }^{1,3}$, Huanhu Zhu ${ }^{1}$, Chongwu $\mathrm{Chi}^{1}$, Mahya Mehrmohamadi ${ }^{2}$, Kejing Deng ${ }^{1}$, Xiaohui $\mathrm{Wu}^{1}$, Tian $\mathrm{Xu}^{1,2}$

${ }^{1}$ State Key Laboratory of Genetic Engineering and National Center for International Research of Development and Disease, Collaborative Innovation Center for Genetics and Development, Fudan-Yale Biomedical Research Center, Institute of Developmental Biology and Molecular Medicine, School of Life Sciences, Children's Hospital, Fudan University, Shanghai 200433, China; ${ }^{2}$ Howard Hughes Medical Institute, Department of Genetics, Yale University School of Medicine, New Haven, CT 06536, USA; ${ }^{3}$ Current address: State Key Laboratory of Dairy Biotechnology, Dairy Research Institute, Bright Dairy \& Food Co., Ltd. Bldg. 2, No.1518, West Jiangchang Road, Shanghai 200436, China

Correspondence: Tian $\mathrm{Xu}^{\mathrm{a}}$, Xiaohui $\mathrm{Wu}^{\mathrm{b}}$

${ }^{a}$ E-mail: tian.xu@yale.edu

${ }^{\text {b}}$ E-mail: xiaohui_wu@fudan.edu.cn

\section{References}

1 Camilleri M. Gastroenterology 2006; 131:640-658.

2 Karhunen LJ, Juvonen KR, Huotari A, et al. Regul Pept 2008; 149:7078.

3 Nicholl C, Polak J, Bloom S. Annu Rev Nutr 1985; 5:213-239.

4 Baker KD, Thummel CS. Cell Metab 2007; 6:257-266.

5 Amanda D, Kathleen B, Ruthann N. Peptides 2002; 23:1953-1957.

6 Peller CR, Bacon EM, Bucheger JA, et al. J Insect Physiol 2009; 55:834-839.

7 Zeng C, Justice NJ, Abdelilah S, et al. Proc Natl Acad Sci USA 2008; 95:10637-10642.

8 Milán M, Diaz-Benjumea FJ, Cohen SM. Genes Dev 1998; 12:29122920.

9 Shoresh M, Orgad S, Shmueli O, et al. Genetics 1998; 150:283-299.

10 Bach I. Mech Dev 2000; 91:5-17.

(Supplementary information is linked to the online version of the paper on the Cell Research website.) 